
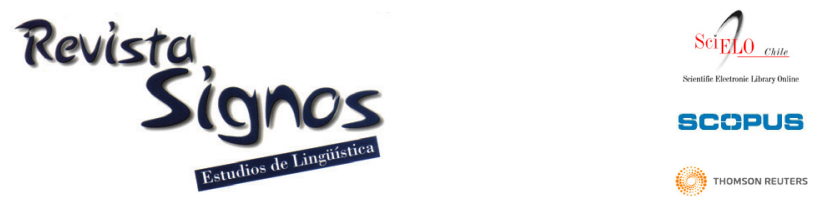

\title{
Léxico Generativo y aplicaciones lexicográficas: Los nombres concretos del dominio de la medicina en el DRAE* $^{*}$
}

\author{
Generative lexicon and lexicographic applications: The medical \\ concrete nouns in the dictionary of the Real Academia Española
}

\author{
Marina Berri \\ Universidad Nacional de General Sarmiento \\ ARgENTINA \\ marinaberri@gmail.com
}

Recibido: 12-II-2012 / Aceptado: 22-X-2012

\section{Resumen}

El presente artículo tiene por objetivo describir las representaciones lexicográficas que ofrece el Diccionario de la Real Academia Española de los nombres concretos del ámbito de la medicina a partir de los valores de la estructura de qualia propuestos en Pustejovsky (1995). Desde una perspectiva que considera la bidireccionalidad entre lingüística teórica y lingüística aplicada, la hipótesis sostenida es que, desde el punto de vista de la aplicación lexicográfica, la estructura de qualia constituye una herramienta que no solo proporciona elementos necesarios para analizar la generación de sentidos, sino que también es una herramienta heurística adecuada para evaluar el grado de sistematicidad y pertinencia de las definiciones lexicográficas. Por otra parte, la estructura de qualia, el concepto de tipo semántico y la noción de quale dominante permiten comenzar a desarrollar una clasificación de nombres concretos a partir del análisis lexicográfico, que permitirá mejorar y homogeneizar su tratamiento lexicográfico. Para realizar el análisis se extrajeron automáticamente del DRAE nombres con las marcas 'medicina' o 'anatomía' y se clasificaron según la ontología UMLS. Luego se analizó qué valores de la estructura de qualia capturan las definiciones y el modo en que lo hacen, en especial las expresiones que se utilizan para dar cuenta de ellos, el orden en que los manifiestan y la parte de la definición en la que los expresan (hiperónimo o diferencia específica). Se comprueba así que la estructura de qualia y la clasificación de nombres por tipo semántico pueden contribuir a la sistematicidad de la lexicografía en lo que hace a los nombres concretos del dominio de la medicina. 
Palabras Clave: Estructura de qualia, nombre concreto, tipo natural, artefactoherramienta, quale dominante.

\begin{abstract}
The aim of this paper is to describe the definitions of concrete medical nouns that appear in the dictionary of the Real Academia Española (Diccionario de la Real Academia Española, henceforth DRAE), using qualia structure (Pustejovsky, 1995). From a point of view that considers the bidirectionality between theoretical and applied linguistics, we argue that qualia structure is a tool that not only provides with the necessary elements to analyze sense generation, but it provides a heuristic tool that can be used to analyze the degree of systematicity and the adequacy of lexicographical definitions. Furthermore, the qualia structure, the semantic type concept, and the idea of dominant quale allow us to start developing a classification of concrete nouns from a lexicographical analysis, which will improve their lexicographical treatment. Concrete nouns marked as medicina (medicine) or anatomía (anatomy) were automatically extracted from DRAE and were classified according to the UMLS ontology. Then, the definitions were analyzed to determine what qualia structure were present in them and in which way, particularly, what expressions were used to refer to these values, what order was used to express them, and in what part of the definition was used to express them (hypernym or specific difference) From these findings, we argue that qualia structure and the classification of nouns based on their semantic type can contribute to lexicographical systematicity in the concrete medical nouns.
\end{abstract}

Key Words: Qualia structure, concrete noun, natural type, artifact-tool, dominant quale.

\title{
INTRODUCCIÓN
}

La teoría del Léxico Generativo (Pustejovsky, 1995, 2011) se encuentra centrada en explicar la polisemia en contexto y el uso creativo de las palabras. Para lograr este objetivo, en este modelo se propone una estructura semántica infraespecificada, que cuenta con cuatro niveles de representación. El nivel de la estructura argumental especifica el número y la naturaleza de los argumentos del predicado, la estructura eventiva define el tipo de evento de una expresión y sus estructuras subeventivas, la estructura de herencia léxica especifica cómo el tipo que posee una palabra se relaciona con otros tipos en una red y, finalmente, la estructura de qualia especifica la fuerza predicativa de un ítem léxico. Por otra parte, se postulan mecanismos que permiten explicar los distintos sentidos que un ítem léxico genera en contexto. En la primera versión del modelo (Pustejovsky, 1995) los mecanismos son la coerción de tipos, el ligamiento selectivo y la co-composición, luego (Pustejovsky, 2011) el ligamiento selectivo, la acomodación, y la coerción de tipos, para la que se proponen las subclases explotación e introducción. 
Esta teoría ha sido utilizada especialmente en aplicaciones de semántica y lexicografía computacional. En particular, ha posibilitado el desarrollo de varias ontologías, como CoreLex (Buitelaar, 1998) y SIMPLE (Busa, Calzorali \& Lenci, 2001) y, en el ámbito del español, se ha aplicado al procesamiento computacional del lenguaje (Climent, 1998; Alonso, Bel \& Pedersen, 2011) y a la representación de la polisemia (Martí, 2003; Adelstein, 2008). A pesar de los trabajos que existen en semántica y lexicografía computacional, la teoría ha sido escasamente aplicada a la confección y análisis de diccionarios de lengua del español. A excepción de los recientes trabajos de Battaner (2010), quien estudia las metáforas basadas en la estructura de qualia y el uso de la marca 'figurado' en diccionarios, y de Batiukova (2009a), que destaca el vínculo entre la estructura de qualia y las definiciones de tipo descriptivo, genético y teleológico, este marco teórico no ha sido desarrollado en lo que respecta a la evaluación y mejora de obras lexicográficas del español. En este artículo me propongo analizar las definiciones que ofrece el Diccionario de la Real Academia Española (de aquí en más, DRAE) de los nombres concretos pertenecientes al ámbito de la medicina a partir de los valores de la estructura de qualia propuestos en Pustejovsky (1995) para comprobar si esta estructura puede contribuir al análisis y mejora de esas definiciones.

A partir de una perspectiva fundada en la bidireccionalidad entre lingüística teórica y aplicada, la hipótesis que sostengo es que la estructura de qualia no solo proporciona elementos necesarios para explicar la generación de sentidos, sino que puede ser empleada como una herramienta heurística que permite evaluar y mejorar las representaciones lexicográficas, a través de la identificación de tendencias en las definiciones. Como señala Batiukova (2009a) un enfoque teórico sólido proporciona coherencia a la obra lexicográfica y, a la vez, la aplicación de una teoría a grandes corpus de datos contribuye a probar la validez de esa teoría. Para cumplir el objetivo mencionado, se analizaron las definiciones lexicográficas de nombres que en el DRAE se marcan como pertenecientes al dominio de la medicina y la anatomía. Los nombres así marcados corresponden a dos clases de tipos semánticos diferentes: los tipos artefacto-herramienta y los tipos naturales.

En la misma línea que Battaner (2010), propongo entonces considerar a la estructura de qualia como un instrumento teórico que permite mejorar las representaciones lexicográficas y el tipo de información que incluyen. Si, como afirma Moravcsik (2001) la estructura de qualia representa 'la anatomía interna del significado léxico', esta herramienta teórica puede hacer aportes útiles a la lexicografía. Por otra parte, la estructura de qualia y el concepto de quale dominante permitirían comenzar a caracterizar los tipos naturales y los artefactos-herramientas en el ámbito de los nombres concretos de la medicina a partir del análisis lexicográfico. El análisis busca también determinar qué información mínima debe representarse según el tipo de nombre. Esta información mínima debería estar presente en todos los diccionarios, mientras que otras informaciones podrían seleccionarse de acuerdo al tipo de usuario 
y diccionario. Por ejemplo, mientras que un diccionario destinado a los primeros años de la enseñanza escolar puede incluir únicamente la información mínima, un diccionario destinado a la enseñanza de los últimos años del colegio puede incorporar otras informaciones, además de aquellas imprescindibles.

A continuación presentaré la metodología que se utilizó para llevar a cabo el trabajo. Luego expondré los resultados del análisis de los qualia en las definiciones y realizaré una síntesis acerca de la relación que existe entre determinados qualia y determinados tipos y subtipos de nombres y cómo esta relación puede contribuir a sistematizar la compilación de definiciones. Finalmente presentaré unas breves conclusiones.

\section{Marco de referencia}

En Thought and language, Moravcsik (1990) sostiene que la primera función del lenguaje es articular el pensamiento y realiza una propuesta de representación semántica que se basa en la idea de que los humanos se caracterizan por buscar explicaciones y que este hecho se refleja en la estructura semántica de las palabras. Los significados serían, por lo tanto, patrones explicativos que permitirían dar cuenta de cómo y por qué algunos elementos caen dentro de la extensión de una palabra dada. En este sentido, explicar algo implicaría postular sus propiedades - propiedades que se encuentran relacionadas de una manera específica- y ordenar elementos en grupos según sus similaridades. De aquí se desprende la relevancia de la clasificación en tipos y subtipos tanto desde el punto de vista teórico como del aplicado, ya que el tipo proporcionaría una primera caracterización en términos de las propiedades que incluye una cierta unidad.

Moravcsik (1990) afirma que existe una correlación entre la cognición y la semántica léxica, dado que los conceptos explicativos de la cognición son sentidos potenciales de las palabras: la estructura misma del significado sería la de un esquema explicativo. Según este autor, el conocimiento de algunas propiedades clave que está involucrado en los usos explicativos es parte de la competencia semántica. Moravcsik (1990) determina así cuatro factores explicativos que conforman el esquema del significado. Estos factores, retomados luego por Pustejovsky (1995), son el resultado de la influencia del punto de vista del agente y no son aleatorios. El primer factor es el factor material, que se fija de acuerdo a cómo está constituido el objeto designado y determina la categoría a la que pertenece un ítem léxico. El factor estructural establece cómo se diferencia un elemento de aquellos que pertenecen a la misma categoría. Estos dos factores conforman la estructura de cualquier ítem léxico. En cambio, el factor funcional y el factor agentivo pueden o no encontrarse presentes en la estructura de una palabra. El factor funcional especifica qué función tiene lo designado por una unidad. El factor agentivo se vincula con el modo en que un objeto ha sido creado y por lo tanto es propio de los artefactos, que son entidades creadas, y 
no de los tipos naturales. Por último, cabe señalar que en las estructuras de algunas expresiones hay un factor dominante que determina el contenido de los otros factores (Moravsick, 1990; Battaner, 2010). Este factor dominante determina en gran parte la adecuación de las definiciones lexicográficas. De acuerdo con Batiukova (2009a: 238): "la prominencia de distintos roles de la EQ para la caracterización de diferentes clases de unidades léxicas determina la existencia de un sistema de tipos". Los tipos naturales están basados en los roles formal y constitutivo, los unificados o funcionales corresponden a los artefactos-herramientas y tienen valores en sus qualia agentivo y télico, además de los valores de los qualia constitutivo y formal. En esta misma línea, Lenci (2001) afirma que los ítems léxicos que son tipos unificados forman parte de diferentes clases en relación con diferentes dimensiones de significado y que cada una de estas dimensiones está especificada en términos de un quale.

El modelo del Léxico Generativo (Pustejovsky, 1995, 2001; también para el español Adelstein, 2008; Batiukova, 2009a, 2009b; De Miguel, 2009 y; específicamente en relación con la lexicografía, Battaner, 2008) retoma los factores de Moravcsik y los integra en la estructura semántica de los ítems léxicos, en el nivel de la estructura de qualia (EQ). La EQ representa los atributos del referente de un ítem léxico según cuatro modos de explicación: formal $(\mathrm{QF})$, constitutivo $(\mathrm{QC})$, télico $(\mathrm{QT})$ y agentivo (QA). En el QF se ofrece información sobre aquello que distingue a la entidad designada en un dominio más amplio, así como sobre su forma, orientación, magnitud, color, posición y dimensiones. Puede considerarse que la consistencia, una de las propiedades de las entidades físicas que refiere a la plasticidad según Frawley (1992), también es parte del QF. En el QC se especifica la información relativa al todo al que pertenece o a las partes que conforman el objeto denotado, además del material del que está constituido y su peso. Finalmente en el QT se representan eventos asociados con sus funciones y en el QA se detalla el proceso que le dio origen. Siguiendo a Adelstein (2008), me referiré a los distintos valores de los qualia como subqualia. Así, el QF consta de distintos subqualia, el subquale ISA, que especifica el tipo de entidad, el subquale forma, el subquale dimensión, el subquale posición, etc.

En relación con la representación lexicográfica, es importante señalar que el QF da cuenta de la relación ISA, que generalmente se captura en las definiciones mediante un hiperónimo. Sin embargo, cabe realizar sobre estos conceptos algunas precisiones que resultan fundamentales para la descripción aquí propuesta. Hiperónimos como 'aparato' o 'instrumento' indican que una entidad tiene el tipo OBJETO FÍSICO y es además un artefacto-herramienta, es decir, tiene quale télico: posee una función a la que el hiperónimo 'instrumento' alude pero no especifica. En cambio otros hiperónimos como 'anestésico' dan cuenta de la relación ISA (un anestésico es una sustancia) y del quale télico, pero de una manera más granular que el hiperónimo 'instrumento', ya que especifica su función ('producir la pérdida de la sensibilidad'). Otros hiperónimos como 'sonda', empleado en la definición de 'catéter', dan cuenta del quale formal en término de la relación ISA (un catéter es una sonda y una sonda es un 
objeto físico) pero al mismo tiempo también de otro subquale del QF: el subquale forma ('tubular'). Los hiperónimos pueden, por lo tanto, dar informaciones con distinto grado de granularidad de los valores de la estructura de qualia y pueden especificar o no distintos subqualia.

Busa, Calzolari, Lenci y Pustejovsky (2001) postulan la estructura de qualia extendida, en la que identifican subtipos de cada uno de los qualia. Distinguen entre tipos funcionales fuertes y débiles. Mientras que los primeros crean tipos nuevos, los segundos agregan información al tipo sin cambiar su naturaleza. La introducción de la EQ extendida está motivada, según los autores, porque ítems léxicos como honey producer ('apicultor') y bee ('abeja'), a pesar de tener un QT, se comportan de manera diferente. Así, mientras que es posible actualizar en contexto el QT de honey producer, que es un QT fuerte, no es posible actualizar el QT de bee, que es un QT débil:

a. an old honey producer

'a person who is old and who produces honey'

'a person who has been producing honey for a long time'

b. an old bee

'a bee which is old'

\# 'a bee which has been producing honey for a long time'

Según Busa, Calzolari, Lenci y Pustejovsky (2001), la información que concierne al QT de bee, que denominan quale de actividad típica, puede ser codificada de manera opcional, ya que a partir de su combinatoria el nombre muestra que es un tipo natural. Sin embargo, la información del QT télico de honey producer debe ser codificada de manera obligatoria, ya que su combinatoria muestra que el nombre es un tipo unificado.

La EQ es extremadamente relevante para el análisis lexicográfico, dado que permite, junto con los mecanismos de generación de significado, explicar distintos tipos de polisemia lógica y caracterizar los sentidos posibles de una voz. Siguiendo los trabajos de Battaner $(2008,2010)$, quien estudia las metáforas basadas en la estructura de qualia y el uso de la marca 'figurado' en diccionarios, y de Batiukova (2009a), que destaca el vínculo entre la estructura de qualia y las definiciones de tipo descriptivo, genético y teleológico, el análisis aquí presentado se propone pensar a la estructura de qualia como un modo de mejorar la compilación de las definiciones lexicográficas, redactadas generalmente de modo intuitivo.

\section{Marco metodológico}

Para realizar este análisis se ha tomado un corpus de aproximadamente $400^{1}$ nombres concretos que llevan en el DRAE las marcas 'medicina' o 'anatomía'. La extracción de los nombres se realizó a partir de búsquedas digitalizadas en la versión 
en CD-Rom del DRAE a través del menú 'consulta avanzada'. Luego, los nombres se clasificaron según la ontología de la UMLS (Unified Medical Language System) ${ }^{2}$. Esta ontología cuenta con una red semántica y un metatesauro. La red semántica, compuesta por 134 tipos y 54 clases de relaciones, provee una categorización consistente de los conceptos del metatesauro, que incorpora 60 vocabularios y casi 900.000 conceptos del dominio de la biomedicina (McGray, 2003). El metatesauro contiene clasificados los términos en los tipos de la red semántica, es decir, la clasificación de la mayoría de los términos ha sido ya realizada por especialistas. Para los términos que no figuran en el metatesauro se ha consultado a un especialista en medicina, quien supervisó su clasificación ${ }^{3}$. En la Figura 1 reproduzco el fragmento de la red semántica que es pertinente para este trabajo, es decir, aquellos tipos que dependen del tipo ENTIDAD FÍSICA y los nombres cuyas definiciones fueron analizadas:

\section{A1. Organismo (...)}

\section{A2. Estructura anatómica}

\section{A2. 1. Estructura embrional}

\section{A2.2. Anormalidad anatómica (...)}

\section{A2.3. Estructura anatómica completa}

\section{A2.3.1. Parte del cuerpo, órgano o componente de un}

órgano: acromion, albugineo, almohadilla, alveario, alveolo, amígdala, amígdala faríngea, amígdala lingual, amígdala palatina, antepié, aorta, apéndice cecal, apófisis, arco alveolar, areola, aritenoides, arteria celiaca, arteria coronaria, arteria emulgente, arteria ranina, arteria subclavia, astas de la médula, astrágalo, atlas, auricula, bazo, biceps, bóveda craneal, bronquio, bronquiolo, bulbo piloso, bulbo raquideo, caja del tímpano, calcáneo, canal torácico, capilar, carótida, cerebelo, cerebro, clavícula, cóccix, colon, conducto arterioso, conducto auditivo externo, conducto cístico, conducto deferente, conducto hepático, conducto inguinal, conducto raquideo, conjuntivo, corazón, córnea, cornete, coroides, corona, cráneo, cricoides, cristalino, cúbito, deltoides, diáfisis, diafragma, disco intervertebral, duodeno, duramadre, emuntorio, encéfalo, epicanto, epididimo, epifisis, epiglotis, esclerótico, escroto, esfinter, espina, esplenio, esófago, espóndilo, esternón, estómago, estribo, falange, faringe, folículo, fóvea, funiculo, ganchudo, ganglio linfático, ganglio nervioso, glándula, higado, himen, hipocampo, hipocondrio, bipófisis, hipotálamo, hueso cuadrado, bueso cuboides, hueso cuneiforme, bueso esfenoides, bueso escafoides, hueso etmoides, hueso frontal, hueso bioides, hueso innominado, bueso intermaxilar, hueso maxilar, hueso occipital, hueso orbital, hueso parietal, hueso piramidal, bueso plano, bueso sacro, hueso temporal, búmero, ilion, intestino, intestino, intestino ciego, intestino delgado, intestino grueso, isquion, laberinto, laringe, lenticular, ligamento, mamila, martillo, masetero, mediopié, médula espinal, médula oblonga, membrana mucosa, membrana pituitaria, membrana serosa, meninge, metacarpo, metatarso, mucronato, músculo, músculo 
braquial, músculo complexo, músculo esternocleidomastoideo, músculo estriado, músculo femoral, músculo gemelo, músculo glúteo, músculo

\section{A. Objeto físico}

lumbrical, músculo oblicuo, músculo recto, músculo redondo, músculo sartorio, músculo serrato, músculo subescapular, músculo vasto, nervio, nervio auditivo, nervio ciático, nervio óptico, nervio vago, neumogástrico, neuroepitelio, notocordio, oído, oído interno, oído medio, omóplato, ovario, paladar blando, paladar duro, palatino, páncreas, panículo, papila, parótida, pectíneo, pelvis, pene, peñasco, pericardio, pericráneo, periodonto, periostio, peritoneo, peroné, piamadre, pisiforme, pleura, plexo, plexo sacro, plexo solar, prepucio, próstata, protuberancia cerebral, pubis, puente de Varolio, pulpa dentaria, pupila, retina, riñón, rótula, sóleo, sustancia blanca, sustancia gris, tálamo óptico, tarso, tendón, tendón de Aquiles, testículo, timo, tiroides, trapecio, trapezoide, triceps, triceps braquial, triceps espinal, triceps femoral, trompa de Eustaquio, trompas de Falopio, tronco braquiocefálico, túnica úvea, unciforme, unguis, uréter, uretra, úvula, vagina, válvula, válvula tricúspide, velo del paladar, vena ácigos, vena basílica, vena cardíaca vena cava, vena cefálica, vena emulgente, vena porta, vena ranina, vena safena, vena subclavia, vena yugular, ventrículo, vértebra, vesícula aérea, vesícula biliar, vesícula ovárica, vesícula seminal, vómer, yeyuno, yunque

\section{A2.3.2. Tejido}

\section{A2.3.3. Célula}

A2.3.4. Componente de la célula

\section{A2.3.5. Gen o genoma}

\section{A3. Objeto manufacturado}

A3.1. Dispositivo médico: algalia, aparato, astigmómetro, bioprótesis, bisturí, bordón, botador, capellina, cardiógrafo, carrilete, catéter, cauterio, cauterio actual, criocauterio, depresor, desfibrilador, ductor, enema ('aparato'), erina, escalpelo, escáner, escarificador, esfigmógrafo, espéculo, espirómetro, estetoscopio, estilete, férula, fonendoscopio, fórceps, fronda, galápago, gastroscopio, hematocrito, hemodializador, implante, inhalador, lanceta, laparoscopio, laringoscopio, lechino, legra, lengüeta, ligadura, longuetas, mandril, monóculo, moxa, neurótomo, otoscopio, pesario, pildorero, plesimetro, prótesis, raquitomo, rectoscopio, respirador, sajador, tenáculo, tensiómetro, tienta, tirafondo, trépano, valva, ventosa, vía

A3.2. Dispositivo de investigación

A3.3. Droga en presentación clínica 


\section{A4. Sustancia}

A4.1. Sustancia química

A4.1.1. Sustancia vista desde el punto de vista funcional

A4.1.1.1. Sustancia farmacológica: aguardiente alemán, alcoholado, alcoholaturo, alexifármaco, anfetamina, angélico, anodino, ansiolitico, antiafrodisiaco, antialérgico, antiartrítico, antiasmático, anticongestivo, antiemético, antiespasmódico, antiflogistico, antihelmíntico, antibidrópico, antibipertensivo, antibistérico, antiinflamatorio, antipirético, antipútrido, antirrábico, antirreumático, antiséptico, antitérmico, antitetánico, antitusígeno, aperitivo, aspirina, betabloqueante, calmante, carminativo, caustico, cerasiote, cerebrina, citostático, cloromicetina constrictor, correctivo, depurativo, derivativo, desopilativo, diacodión, diascordio, digestivo, dormitivo, drástico, electuario, emenagogo, emetina, enema ('medicamento'), estovaina, evacuativo, expectorante, filonio, fundente, hemostático, insulina, lactuario, melito, metadona, minorativo, mitridato, pildora alefangina, quermes, reconstituyente, relajante, repercusivo, revulsivo, revulsorio, salipirina, salol, sinapismo, solutivo, tenifugo, tridacio, vasoconstrictor, vasodilatador, vermifugo, vomipurgante, vomitivo, vulnerario

A4.1.1.2. Material biomédico o dental

A4.1.1.3. Sustancia biológicamente activa

A4.1.1.4. Indicador, reactivo o ayuda para el diagnóstico

A4.1.1.5. Sustancia venenosa o peligrosa

A4.1.2. Sustancia vista desde el punto de vista estructural (...)

A4.2. Sustancia del cuerpo

A4.3. Comida

Figura 1. Los nombres extraídos del DRAE en la ontología UMLS.

Los tres tipos analizados son PARTE DEL CUERPO, ÓRGANO O COMPONENTE DE UN ÓRGANO (DE AQUÍ EN MÁS PARTE DEL CUERPO), INSTRUMENTO MÉDICO Y SUSTANCIA FARMACOLÓGICA y se han seleccionado por ser los más frecuentes en el DRAE. Otros tipos, como MATERIAL BIOMÉDICO O DENTAL Y TEJIDO, corresponden a nombres que el DRAE recoge escasamente o bien a nombres que no llevan las marcas 'medicina' o 'anatomía'. Por otra parte, el tipo parte del cuerpo de esta ontología corresponde a nombres que son de tipo natural, y los tipos INSTRUMENTO MÉDICO Y SUSTANCIA FARMACOLÓGICA corresponden al tipo unificado artefacto- 
herramienta. Esto permite poner a prueba la relevancia de la distinción entre tipos naturales y artefactos-herramientas desde el punto de vista lexicográfico.

Una vez clasificados los nombres se procedió a extraer sus definiciones. Este conjunto de definiciones constituye el corpus de análisis y consta de 262 definiciones de PARTES DEL CUERPO, 65 definiciones de INSTRUMENTOS MÉDICOS y 89 definiciones de SUSTANCIAS FARMACOLÓGICAS, es decir, un total de 416 definiciones. A partir de estos datos, se determinó qué valores de la EQ capturan las definiciones y se estudió el modo en que lo hacen, en especial las expresiones que se utilizan para dar cuenta de ellos y la parte de la definición en la que los expresan (hiperónimo o diferencia específica). Para establecer y reconocer los valores de los qualia de las unidades se establecieron las siguientes paráfrasis de la Figura 2:

Para el subqualia ISA del QF $\rightarrow \mathrm{X}$ es un (tipo de) Y (un plesimetro es un tipo de instrumento)

Para el QC $\rightarrow \mathrm{X}$ es parte de Y / X está constituido por Y (el acromion es parte del omóplato / el mediopié está constituido por el escafoides, el cuboides y las cuñas / el ligamento está compuesto por fibras)

Para el QT fuerte $\rightarrow$ X falla / estimular X (fallar el corazón / estimular la tiroides)

Para el QT débil $\rightarrow$ X cumple la función de Y (el epicanto cumple la función de recubrir el ángulo interno del ojo)

Para el QA $\rightarrow \mathrm{X}$ se obtiene de manera Y (la insulina se obtiene de manera natural y artificial)

Figura 2. Paráfrasis que permiten reconocer los qualia.

Se extrajeron así tendencias en las definiciones, que permitieron observar en qué porcentaje de casos de cada tipo de nombres se ofrece información acerca de qué qualia. Para evaluar la sistematicidad, se tuvo en cuenta si las definiciones de las diferentes clases de nombres se formulan de modo similar o si presentan diferencias por razones semánticas o bien por la falta de un criterio sistemático que permita compilar adecuadamente los ítems léxicos. Finalmente, se elaboró una pequeña encuesta completada por 20 informantes ${ }^{4}$, mediante la que se buscó establecer si las definiciones que no se adaptaban a las tendencias generales del diccionario resultaban para los informantes menos claras. En esta encuesta los informantes señalaron aquellas definiciones que no les parecía claras de una lista y, de otra lista diferente, marcaron aquellas definiciones que no les permitían entender para qué servía aquello que designaba la voz definida. Por último, indicaron qué definición preferirían de dos definiciones en que se presentaba la misma información pero en distinto orden. 


\section{Resultados}

En este apartado presento los resultados del análisis según los cuatro qualia. En los artículos del diccionario que figuran como ejemplo subrayo la parte que analizo de la definición y coloco entre corchetes a qué quale y/o subquale corresponde.

\subsection{Quale formal}

La noción de quale formal permite identificar en el DRAE dos clases de definiciones diferentes según el nombre corresponda a la clase de los tipos naturales o a la de los artefactos-herramienta. En las definiciones de los tipos naturales -los nombres del TIPO PARTE DEL CUERPO- el hiperónimo suele dar cuenta de otros subqualia del QF, además del subquale ISA que indica a qué tipo pertenece la unidad, o bien dar cuenta de la relación ISA también en términos de esos otros subqualia, mediante el uso de hiperónimos que codifican la relación ISA a través de los subqualia forma, color y/o consistencia. Por ejemplo, para los nombres 'conducto auditivo externo' y 'bulbo piloso' se utilizan respectivamente los hiperónimos 'tubo' y 'abultamiento', que especifican el subquale forma del QF: un tubo es un 'objeto cilíndrico alargado y hueco' y un 'abultamiento' es una 'parte saliente'. En los casos en que el hiperónimo mismo no da cuenta del subquale forma, esto se especifica en la diferencia específica, como se observa en los ejemplos (1) y (2):

1. Disco intervertebra1.1. m. Anat. Formación [QF, ISA] fibrosa con forma de disco $[\mathrm{QF}$, forma], entre dos vértebras, en cuyo interior hay una masa pulposa.

2. Funículo. (Del lat. funicŭlus, cuerda).1. m. Anat. Estructura [QF, ISA] de unión en forma de cordón [QF, forma], como los cordones nerviosos de la médula espinal.

Otro de los subqualia del QF que se codifica en las definiciones de las partes del cuerpo es el subquale posición, como se observa en el ejemplo (3). Este subquale se codifica en el 50\% de las definiciones de PARTES DEL CUERPO, lo que da cuenta de su relevancia para definir esta clase de nombres:

3. Epidídimo.1. m. Anat. Órgano [QF, ISA] con aspecto de madeja u ovillo [QF, forma], situado sobre cada uno de los testículos [QF, posición] y constituido por la reunión de los vasos seminíferos.

Esta tendencia a dar cuenta de otros subqualia del QF distintos de la relación ISA se cumple en un $93 \%$ de las definiciones de las PARTES DEL CUERPO. La mayoría de las excepciones la constituyen las glándulas y los órganos que, como explicaré en el subapartado 3.3, tienen un comportamiento particular en contexto. El ejemplo (4) constituye un ejemplo de estas definiciones:

4. Glándula. (Del lat. glandŭla).1. f. Anat. Órgano [QF: ISA] cuya función es producir una secreción que puede verterse a través de la piel o de las mucosas, 
como las glándulas salivales y sudoríparas, o al torrente sanguíneo, como el tiroides.

En este sentido, el análisis muestra que el QF de los tipos naturales se codifica con un alto grado de granularidad, dado que además de codificar la relación ISA los hiperónimos y la diferencia específica codifican otros subqualia del QF, como la forma, el color y la posición. Esta tendencia se invierte en el caso de las voces que designan dispositivos médicos. Para las voces que corresponden a la clase artefactoherramienta de los instrumentos médicos se emplean en la mayoría de los casos $(70 \%)$, los hiperónimos 'aparato', 'instrumento' o 'artificio' tal como se observa en el ejemplo (5). Estos hiperónimos, si bien codifican la relación ISA, ya que describen las voces definidas como tipos unificados de la clase OBJETO FÍSICO_HERRAMIENTA, no precisan los demás subqualia del QF, como la forma, magnitud, dimensiones, posición, etc. Además, estos hiperónimos hacen referencia al QT, ya que un aparato es un objeto físico que tiene una función. Es decir, los hiperónimos dan cuenta del hecho de que se trata de un tipo unificado, ya que aluden a su materialidad (son objetos físicos) y al hecho de que tienen una función:

5. Espirómetro (...).1. m. Med. Aparato [QF: ISA] para medir la capacidad respiratoria del pulmón.

De las definiciones que se realizan mediante los hiperónimos mencionados, solo en un $15 \%$ de los casos se agrega información en la diferencia específica que detalla los demás subqualia del QF, como la forma y la magnitud. En la mayoría de los casos, entonces, estas dimensiones quedan sin precisar. Esto es importante porque pone de manifiesto que estos nombres pueden definirse con un mínimo de información relativa al QF, es decir, solo a través de la especificación del subqualia ISA. Hay, sin embargo, algunas excepciones a esta tendencia, que ilustro con la definición de 'fórceps' del ejemplo (6):

6. Fórceps (...) 1. m. Med. Instrumento $[\mathrm{QF}: \mathrm{ISA}]$ en forma de tenaza $[\mathrm{QF}$ : forma], que se usa para la extracción de las criaturas en los partos difíciles.

Una tendencia similar se observa en los nombres del tipo SUSTANCIA FARMACOLÓGICA que son también artefactos-herramienta. En un 30\% de las definiciones no se proporciona información relativa al QF aunque en muchos casos, de acuerdo con la ontología, estas voces designan únicamente sustancias. Se trata de las voces que son adjetivos que se han sustantivado, como 'antialérgico':

7. Antialérgico, ca.1. adj. Med. Que combate o previene una reacción alérgica. U. t. c. s. m.

Los hiperónimos más frecuentes que se emplean para definir las voces que corresponden a estos tipos son 'medicamento' y sus sinónimos 'medicina', 'droga' y 'remedio', que se usan en el 60\% de las definiciones que incluyen un hiperónimo y dan cuenta, además del subquale ISA del QF, del QT 'curar' y del QA ('creado 
artificialmente'). Del resto de los hiperónimos, que cubre el 40\% restante de las definiciones, la mitad especifica de manera más granular el QT. Tal es el caso de 'purgante' y 'antibiótico', en donde el QT está especificado más granularmente que en 'medicamento'. El resto de los hiperónimos sí codifica la relación ISA en términos de otros valores, por ejemplo, 'jarabe' y ‘sólido'. Dado que se trata de sustancias, es lógico que las definiciones no den información acerca de su forma, posición o dimensiones. Los únicos valores posibles, el color, y la consistencia están escasamente representados ( $5 \%$ de los casos) en la diferencia específica de algunos nombres. En resumen, puede afirmarse que solo en el $20 \%$ de las definiciones se dan especificaciones de otros subqualia del QF diferentes del subquale ISA o bien se define la relación ISA por medio de esos otros valores.

El análisis del DRAE permite detectar, entonces, dos tendencias distintas. En el caso de los nombres de tipo natural parte del cuerpo, el QF se especifica de manera granular, detallando valores para los distintos subqualia. En cambio, en las definiciones de los INSTRUMENTOS MÉDICOS y las SUSTANCIAS FARMACOLÓGICAS, que son artefactos-herramientas, la especificación del QF es menos granular.

\subsection{Quale constitutivo}

En las PARTES DEL CUERPO, el QC tiene una gran relevancia ya que, como el nombre del tipo lo indica, estos sustantivos designan partes del cuerpo humano (huesos, órganos, etc.). Es por eso que en el inicio del enunciado de la definición o de la diferencia específica se hace referencia al QF, es decir, se da cuenta de las relaciones de meronimia que mantiene con otras unidades, tal como se observa el ejemplo (8):

8. Antepié 1. m. Anat. Parte $[\mathrm{QC}]$ anterior del pie, formada por los cinco metatarsianos y las falanges $[\mathrm{QC}]$ de los dedos correspondientes.

En el 85\% de las definiciones de este tipo hay información sobre el QC, ya que las definiciones incluyen el holónimo, el merónimo o el material del que están constituidas estas entidades. En el caso de de los INSTRUMENTOS MÉDICOS Y DE LAS SUSTANCIAS FARMACOLÓGICAS, en cambio, se proporciona información del QC de los nombres únicamente en un $30 \%$ y un $22 \%$ de las definiciones.

Para la mayoría de los informantes encuestados las definiciones de los tipos naturales que codifican el QF y el QC resulta suficiente. Así, solo 1 de los 20 encuestados señaló que resultaba poco claro el ejemplo (9) que tiene únicamente información del QF y del QC:

9. Bóveda craneal.1. f. Anat. Parte superior e interna del cráneo.

Cuando falta información del QC, como en el caso del ejemplo (10) que no especifica de qué segunda fila se trata, los informantes señalan que la definición es incompleta en un $55 \%$ de los casos: 
10. Trapecio. (Del lat. trapež̆um, y este del gr. $\varrho_{Q} \alpha \pi \dot{\varepsilon} \zeta$ เov). 2. m. Anat. Uno de los huesos del carpo, que en el hombre forma parte de la segunda fila.

En cambio, en los nombres que pertenecen al tipo ARTEFACTOHERRAMIENTA, cuando el QC no se encuentra detallado y el QT sí, la definición no se percibe como incompleta. Así, un único informante marcó como incompleta la definición de 'hematocrito', que no cuenta con información acerca del QC:

11. Hematocrito. (De hemato- y el gr. xœı ós, separado).1. m. Med. Aparato centrifugador que separa las células sanguíneas del plasma para averiguar su proporción relativa y para realizar otros análisis.

Sin embargo, las definiciones de 'alcoholaturo' y 'diacodión', que incorporan el QC pero no especifican la función, más allá de la función general de 'curar' capturada por el hiperónimo 'medicamento', fueron consideradas por el 50\% y el 75\% de los informantes, respectivamente, como incompletas:

12.Diacodión (...) 1. m. Med. Jarabe de adormidera [QC].

13. Alcoholaturo.1. m. Med. Medicamento que se obtiene macerando plantas frescas en alcohol $[\mathrm{QC}]^{6}$.

Cabe señalar que solo el 10\% de los informantes calificó de incompleta la definición de 'antitetánico', que codifica únicamente el QT:

14. Antitetánico, ca.1. adj. Med. Dicho de un medicamento: Empleado contra el tétanos.

La diferencia entre los ejemplos (12) y (13) frente al ejemplo (14) radica en la granularidad de la especificación de los QC y QT. En el caso de 'alcoholaturo', se especifica el QC pero el QT se recoge únicamente mediate el hiperónimo 'medicamento'. En cambio, en el caso de 'antitétánico', el QC e incluso el QF quedan sin especificar pero se detalla el QT al señalar contra qué enfermedad se emplea el medicamento.

Esta mayor relevancia del QC en los nombres de tipo natural y del QT en los nombres artefacto-herramienta también se ve reflejada en el orden. Así, los informantes señalaron en un $80 \%$ de los casos que prefieren que la información relativa al QT se presente antes que la información relativa al QC en la definición de 'salol'. Esta proporción se invierte en 'músculo vasto', en donde en un $80 \%$ de los casos prefieren que la información relativa al QC se presente primero que la relativa al QT.

La comparación entre el QC y QT permite establecer que, mientras el qualia constitutivo es fundamental para el tipo natural PARTE DEL CUERPO no resulta fundamental para artefacto-herramienta de los tipos SUSTANCIA FARMACOLÓGICA e INSTRUMENTO MÉDICO, ya que las definiciones que no lo especifican no se perciben como incompletas. 


\subsection{Quale télico}

En los INSTRUMENTOS MÉDICOS y en las SUSTANCIAS FARMACOLÓGICAS, en el 97\% y 89\% de las definiciones el QT se especifica en la diferencia específica, mediante un adjetivo, una oración de relativo o una subordinada final. En cambio, en los tipos naturales parte del cuerpo el QT se especifica mediante una relativa, adjetivo o subordinada final solo en un $42 \%$ de los casos. La ausencia de la especificación del QT más allá del hiperónimo 'instrumento' afecta la percepción de las definiciones de los artefactos-herramientas, pero no las partes del cuerpo. Así, el $65 \%$ y el $85 \%$ de los informantes calificó de incompletas las definiciones de 'carrilete' y 'galápago', respectivamente, en las que no se especifica el QT, frente a la definición de 'longuetas' que detalla el QT y ninguno señaló como incompleta, y a la definición de 'papila', que corresponde al tipo natural parte del cuerpo y a la que solo el $10 \%$ de los informantes consideró incompleta a pesar de no especificar el QT:

15. Carrilete.1. m. Med. Cierto instrumento quirúrgico usado antiguamente.

16. Galápago 12. m. Med. Tira de lienzo, cuadrilonga, hendida por los dos extremos, sin llegar al medio, y que forma por lo común cuatro ramales.

17. Longuetas. (De luengo).1. f. pl. Med. Tiras de lienzo, ya sencillas, ya dobles o triples, que se aplican en fracturas o amputaciones [QT].

18. Papila 2. f. Anat. Prominencia que forma el nervio óptico en el fondo del ojo y desde donde se extiende la retina.

Asimismo, en el caso de definiciones de las SUSTANCIAS FARMACOLÓGICAS en que el QT debe desprenderse de la morfología o de un término técnico, como en (19) y (20), los informantes indicaron que la definición resulta incompleta. Así, el 75\% de los informantes señaló que 'diacodión' tiene una definición incompleta, dado que la especificación del QT se desprende de la morfología de 'adormidera', y el 85\%, que no le resulta posible establecer con claridad la función de 'emetina', que se desprende del término técnico 'alcaloide':

19. Diacodión (...) 1. m. Med. Jarabe de adormidera.

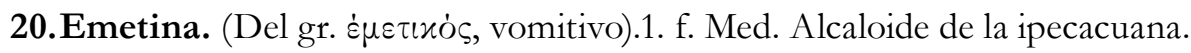

Tanto los nombres analizados que constituyen artefactos-herramientas o tipos naturales tienen un quale télico. Sin embargo, el estatus de este quale es diferente según la clase de nombres. El QT de los artefactos-herramientas es indirecto o de propósito, es decir, en él se expresa el hecho de que los objetos designados son usados por un agente para realizar una actividad (Pustejovsky, 1995). Esto se pone de manifiesto en las definiciones mediante el uso de la voz pasiva, cláusulas de infinitivo o participios pasivos que complementan al hiperónimo e implican la presencia de un agente, tal como se observa en los ejemplos (21-23): 
21. Digestivo 3. m. Med. Medicamento que se aplica para promover y sostener la supuración de las úlceras y heridas [QT].

22.Raquítomo (...) 1. m. Med. Instrumento para abrir el conducto vertebral sin interesar la médula [QT].

23.Depresor 2. m. Med. Instrumento, como el que se aplica a la base de la lengua para dejar libre la cavidad faríngea, usado para deprimir [QT] (|| hundir).

En algunos casos, como en (24), la función no se especifica, pero sí se aclara el dominio. Sin embargo, estas definiciones resultan poco satisfactorias. Así, el 45\% de los informantes señaló que 'botador' presenta una definición incompleta:

24. Botador 6. m. Med. Hierro en forma de escoplillo, dividido en dos dientes o puntas, que usan los dentistas.

Si bien en la definición de los tipos naturales PARTE DEL CUERPO aparece muchas veces la fórmula 'que sirve para', esta se da con mucho menos frecuencia que en la de los artefacto-herramienta y no involucra la presencia implícita de un agente. De hecho, como señala Adelstein (2006), el QT de las partes de los organismos no se corresponde con ninguno de los dos tipos de qualia planteados por Pustejovsky, el QT directo (que implica el propósito que tiene un agente al llevar a cabo un acto) y el QT indirecto (el que presentan los instrumentos, que son utilizados por un agente para realizar algo). De acuerdo con Adelstein (2006: 87), el QT de los organismos se aproxima al QT indirecto, pero estos "presentarían un propósito no volitivo, una funcionalidad típica específica de organismos". Podría pensarse, según esta autora, que los valores del QT de los organismos corresponden al QT de actividad típica presentado en Busa, Calzolari, Lenci y Pustejovsky (2001). El ejemplo (25) ilustra cómo se representa este tipo de quale en el DRAE:

25. Retina (...).1. f. Anat. Membrana interior del ojo, constituida por varias capas de células, que recibe imágenes y las envía al cerebro a través del nervio óptico [QT].

Por otra parte, los QT de los INSTRUMENTOS MÉDICOS y delas SUSTANCIAS FARMACOLÓGICAs son diferentes de los del tipo natural parte del cuerpo en lo que respecta a la posibilidad de actualizarse en contexto. En Busa, Calzolari, Lenci y Pustejovsky (2001) se establece una diferencia entre qualia fuertes y qualia débiles. Mientras que los qualia fuertes conforman tipos unificados, los débiles solo agregan información a los tipos simples. Los QT de los instrumentos son fuertes, y pueden actualizarse en determinados contextos. Por ejemplo, en los siguientes contextos se activa el QT de los artefactos-herramientas, mientras que con la mayoría de los tipos naturales PARTE DEL CUERPO se producen secuencias agramaticales. Las frases (a-d) ilustran este fenómeno: 

a. Falló el escáner.
b. Falló el tensiómetro.
c. * (Le) Falló la pupila.
d. * (Le) Falló el cráneo.

Sin embargo, entre los tipos naturales PARTE DEL CUERPO, algunos de los nombres que designan órganos y glándulas tienen también un QT fuerte, como se desprende de las secuencias (e-g). De hecho, en algunos casos como en (26) la función es casi lo único que se emplea para definir a un órgano:

e. Le falló el corazón.

f. Le fallaron los riñones.

g. Estimular la tiroides.

26. Oído 5. m. Anat. Cada uno de los órganos que sirven para la audición [QT].

En los casos en que la función de un órgano es múltiple, el quale se vuelve débil, lo que indica que, además de poseer una función, para que un quale télico sea fuerte, esta función debe ser también específica:

h. *Le falló el cerebro.

La ausencia de la función en la definición de voces de los órganos parece deberse a un criterio asistemático, ya que se proporcionan a veces para nombres cuyo referente tiene una función compleja (como por ejemplo ‘bazo' del que se dice “que destruye los hematíes caducos y participa en la formación de los linfocitos"), pero no para otros como 'laringe'. Dada la importancia de este quale para los artefactos-herramientas y para la subclase de los órganos y glándulas del tipo PARTE DEL CUERPO, debería haber siempre información relativa a él en las definiciones de este tipo y subtipo de nombres.

El análisis del QT permite detectar irregularidades que afectan la calidad de las definiciones. Compárese por ejemplo las definiciones de 'retina' y 'pupila' en (27) y (28), cuya función se especifica, y de ‘cristalino' en (29), que solo se define por sus QF y QC:

27. Retina (...)1. f. Anat. Membrana interior del ojo, constituida por varias capas de células, que recibe imágenes y las envía al cerebro a través del nervio óptico [QT].

28.Pupila 3. f. Anat. Abertura circular o en forma de rendija de color negro, que el iris del ojo tiene en su parte media y que da paso a la luz [QT]. 
29. Cristalino 4. m. Anat. Cuerpo en forma de lente biconvexa, situado detrás de la pupila del ojo de los vertebrados y de los cefalópodos.

Ruimy, Gola y Monachini (2001) sostienen que determinado valor de la estructura de qualia puede expresarse en una definición indirectamente a través de la clase semántica del hiperónimo. En lo que respecta a las fórmulas de definición, puede afirmarse que mientras que la función en los tipos naturales PARTE DEL CUERPO suele ser capturada por el hiperónimo ('conducto', 'arteria', 'hueso' y 'músculo' son algunos de los hiperónimos más frecuentes), en los INSTRUMENTOS MÉDICOS Y SUSTANCIAS FARMACOLÓGICAS el QT se expresa mediante una subordinada relativa o una cláusula de infinitivo o participio. Este último modo de representar la información permite explicitar y desplegar más granularmente el QT, además de dar cuenta de la presencia de un agente.

\section{4. Quale agentivo}

El quale agentivo es el que tiene menos presencia en el diccionario. Generalmente este quale no se representa en las definiciones debido al hecho de que puede inferirse de la misma definición si se trata de un tipo natural o de un artefacto. Únicamente en un $8 \%$ de las definiciones de las SUSTANCIAS FARMACOLÓGICAS se precisa si se trata de una sustancia natural o artificial. En el caso de los artefactos-herramientas, esta especificación queda capturada por los hiperónimos 'aparato' e 'instrumento'.

\section{Discusión}

El análisis anterior ha demostrado que la estructura de qualia es un instrumento útil para evaluar las definiciones lexicográficas, así como para clasificar los nombres según sean tipos naturales o artefactos-herramientas. Se ha probado que determinados qualia son cruciales para definir ciertos tipos y subtipos de nombres, mientras que otros son menos relevantes. Tener en cuenta los qualia dominantes puede ayudar a obtener diccionarios más sistemáticos y definiciones más adecuadas y fácilmente comprensibles ya que, como se ha observado en varios artículos del DRAE, su ausencia produce definiciones que se perciben como incompletas. A continuación presento una síntesis acerca de qué qualia resultan indispensables para definir tipos y subtipos de nombres, y cómo esta información podría utilizarse en la redacción de definiciones. Sin embargo, esta clasificación constituye una guía que no busca reemplazar el trabajo del lexicógrafo de evaluar cada unidad concreta, sino simplemente agilizar su tarea y contribuir a la revisión de su calidad.

\subsection{Tipos naturales: PARTE DEL CUERPO, ÓRGANO O COMPONENTE DE UN ÓRGANO}

En los tipos naturales los qualia formal y constitutivo son los dominantes. Por lo tanto, las definiciones deberían contar con especificaciones granulares acerca de 
estos qualia. Dada la relevancia de esta clase de información resulta pertinente que sea capturada en el hiperónimo o en la primera parte de la diferencia específica de la definición y que se detallen los distintos subqualia, siguiendo la tendencia general que presentan los diccionarios. En lo que respecta a los subtipos específicos, pueden hacerse las siguientes aclaraciones. A pesar de que, desde el punto de vista de la realidad -y de acuerdo con la ontología- los órganos y las glándulas son partes del cuerpo, el quale télico de estos nombres es fuerte. Por lo tanto, la función debe ocupar un lugar en la definición. Sin embargo, el patrón de definición que resulta más adecuado para dar cuenta de los órganos es el mismo que se utiliza para los otros tipos naturales PARTE DEL CUERPO (un verbo conjugado que denota la función en presente del indicativo) y responde al quale télico de actividad típica.

\subsection{Artefactos-herramienta: INSTRUMENTOS MÉDICOS Y SUSTANCIAS FARMACOLÓGICAS}

El quale télico es el elemento fundamental que permite definir adecuadamente los artefactos-herramientas, ya que es el quale dominante. El uso de un hiperónimo como 'instrumento' o 'aparato' que codifica la relación ISA haciendo referencia a su quale télico parece ser el más acertado y las intuiciones de los lexicógrafos apoyan esta afirmación. Sin embargo, resulta imprescindible hacer mayores especificaciones acerca de la función que cumplen estos instrumentos. El uso de patrones de definición como 'que se usa para' o bien 'usado para' contribuye a especificar el QT y a dar cuenta de que se trata de un quale télico indirecto.

La especificación de la relación ISA y de la función en las definiciones constituye la información mínima requerida para definir este tipo de nombres, ya que de otro modo, como en el caso de 'galápago', las definiciones se perciben como incompletas. En cambio, la especificación del QC de los nombres y de otros subqualia del QF (forma, color, posición, etc.) constituyen informaciones adicionales que pueden incluirse en diccionarios destinados a usuarios adultos, pero de la cual podría prescindirse en diccionarios destinados a los primeros años de escolarización o en diccionarios monolingües destinados a estudiantes de una lengua segunda. Así, de acuerdo con el usuario para el que se diseñe el diccionario pueden pensarse distintas especificaciones del QT. En el ejemplo (26) extraído del DRAE se observan dos tipos de especificaciones: la primera da cuenta de la acción específica de la sustancia, mientras que la segunda da cuenta de la enfermedad o síntoma que combate. De acuerdo con el usuario del diccionario puede incorporarse a la definición una o ambas especificaciones. De este modo para un diccionario escolar bastaría con dar cuenta de la enfermedad que combate, mientras que para un diccionario especializado debería detallarse también la acción específica de la sustancia:

30. Betabloqueante.(De beta1 y bloquear, por unirse a los receptores adrenérgicos de tipo $\beta$, bloqueándolos).1. m. Med. Sustancia que inhibe la acción de la adrenalina en la transmisión nerviosa. Los betabloqueantes se utilizan en el tratamiento de la hipertensión. 


\section{CONCLUSIONES}

Del presente trabajo es posible extraer una serie de conclusiones teóricas y aplicadas. En cuanto a las conclusiones aplicadas, el análisis ha permitido comprobar que la estructura de qualia es una herramienta que permite evaluar la sistematicidad y la pertinencia de las definiciones de un diccionario. En futuros estudios esta descripción puede, por un lado, llevarse a cabo en nombres que no pertenezcan a un dominio de especialidad y, por otro, extenderse al análisis de diccionarios de especialidad. Por otra parte, el análisis resulta un buen modo de establecer cuánta información se desea incluir en las definiciones de un diccionario, ya que permite determinar qué información es más central según qué tipo de nombre. Podría considerarse que las definiciones que incluyen información adicional además de la información central se encuentran más cerca del tipo de definición enciclopédica. Si la cantidad de información enciclopédica que figura en las entradas debe adoptarse sistemáticamente por convención, el empleo de la estructura de qualia ayudaría a delimitar de manera homogénea la cantidad de información que se desea incluir, teniendo en cuenta la especificación de qué qualia y subqualia resultan imprescindibles según qué tipo de nombre. Por último, el trabajo ha demostrado que las clasificaciones nominales más refinadas pueden ser de utilidad para sistematizar y agilizar el trabajo lexicográfico, así como para mejorar sus productos.

En cuanto a las conclusiones de orden teórico, se ha aportado evidencia en relación con la idea de que existen qualia dominantes para distintos tipos y subtipos de nombres. Las definiciones reflejan la prominencia de estos qualia mediante estrategias como la elección del hiperónimo y la falta de información acerca de otros qualia. El análisis que se llevó a cabo permitió llegar a la conclusión de que, si bien no hay fórmulas ni patrones rígidos, en el DRAE se observan ciertas tendencias que permiten representar los valores de la estructura de qualia, así como los diferentes tipos semánticos planteados por el Léxico Generativo. Esto pone de manifiesto que la intuición del lexicógrafo acerca de los rasgos de significado que caracterizan a un ítem léxico coincide, hasta cierto punto, con las observaciones teóricas de Pustejovsky y Moravcsik. De acuerdo con Ruimy et al. (2001: 351):

"The adequacy of qualia relations for capturing key aspects of word meaning becomes apparent when consulting dictionary definitions. The elements of meaning easily map on the dimension(s) expressed via qualia roles. Furtheremore, these relations become particularly crucial for those sense definitions which have an underspecified genus term".

En lo que hace específicamente al modelo del Léxico Generativo, se ha concluido que el hecho de que cierto quale, en particular el quale télico, sea fuerte o débil depende en parte de cuán específico sea su valor. Además, se ha contribuido a la clasificación nominal en el dominio de la medicina a partir de este modelo. 


\section{REFERENCIAS BIBLIOGRÁFICAS}

Adelstein, A. (2006). Semántica especializada y modelización de la entrada léxica de los nombres relacionales. Revista de la Sociedad Argentina de Lingüistica, 1, 71-94.

Adelstein, A. (2008). Unidad léxica y significado especializado: Modelo de representación a partir del nombre relacional madre. Tesis de doctorado, Institut Universitari de Lingüística Aplicada, Barcelona, España.

Alonso, H., Bel, N. \& Pedersen, B. (2011). Identification of sense selection in regular polysemy using shallow features. En B. Pedersen, G. Nešpore \& I. Skadina (Eds.), Proceedings of the 18th Nordic Conference of Computational Linguistics (pp. 1825). Riga, Latvia: Northern European Association for Language Technology.

Batiukova, O. (2009a). Aplicaciones lexicográficas de la teoría del Lexicón Generativo. En E. De Miguel, S. U. Sánchez Jiménez., A. Serradilla Castaño, R. Radulescu \& O. Batiukova (Eds.), Fronteras de un diccionario. Las palabras en movimiento (pp. 233-270). San Millán de la Cogolla: Cilengua.

Batiukova, O. (2009b). La teoría del léxico en los nuevos diccionarios. En E. De Miguel (Ed.), Panorama de la lexicología (pp. 487-516). Barcelona: Ariel.

Battaner, P. (2008). El fenómeno de la polisemia en la lexicografía actual: Otra perspectiva. Revista de Lexicografía, 14, 7-25.

Battaner, P. (2010). La marca figurado como marca de uso. En E. Bernal, J. De Cesaris \& S. Torner (Eds.), Estudis de lexicografia 2003-2005 (pp. 39-60). Barcelona: Institut Universitari de Lingüística Aplicada. Universitat Pompeu Fabra.

Buitelaar, P. (1998). CoreLex: Systematic Polysemy and Underspecification. Tesis doctoral, Brandeis University, Brandeis, Estados Unidos.

Busa, F., Calzolari, N. \& Lenci, A. (2001). Generative Lexicon and the SIMPLE model: Developing Semantic Resources for NLP. En P. Bouillon \& F. Busa (Eds.), The language of word meaning (pp. 333-349). Cambridge: Cambridge University Press.

Busa, F., Calzolari, N., Lenci, A. \& Pustejovsky, J. (2001). Building a semantic lexicon: Structuring and generating concepts. En H. Bunt, R. Musken \& E. Thijsse (Eds.), Computing Meaning (pp. 29-51). Dordrecht: Kluwer Academic Publishers.

Climent, S. (1998). Individuación e Información Parte-Todo. Representación para el procesamiento computacional del lenguaje. Tesis Doctoral, Universidad de Barcelona, Barcelona, España. 
Copestake, A. (1995). The representation of group denoting nouns in a lexical knowledge base. En P. St. Dizier \& E. Viegas (Eds.), Computational Lexical Semantics (pp. 207-230). Cambridge: Cambridge University Press.

De Miguel, E. (2009). La teoría del Lexicón Generativo. En E. De Miguel (Ed.), Panorama de la lexicología (pp. 337-366). Barcelona: Ariel.

Frawley, W. (1992). Linguistic Semantics. Nueva Jersey: Lawrence Erlbaum Associates.

Lenci, A. (2001). Building an Ontology for the Lexicon: Semantic types and word meaning. En P. Jensen \& P. Skadhauge (Eds.), Ontology-Based Interpretation of Noun Phrases. Proceedings of the First International OntoQuery Workshop (pp.103120). Kolding: Department of Business Communication and information Science, University of Southern Denmark.

Martí, M. (2003). Consideraciones sobre la polisemia. En M. Martí Antonín, A. Fernández Monraveta \& G. Vázquez García (Eds.), Lexicografía computacional y semántica (pp. 61-103). Barcelona: Ediciones de la Universidad de Barcelona.

McCray, A. T. (2003). An Upper-level Ontology for the Biomedical Domain. Comparative And Functional Genomics, 4(1), 80-84.

Moravcsik, J. (1990). Thought and language. Londres: Routledge.

Moravcsik, J. (2001). Metaphor, creative understanding and the Generative Lexicon. En P. Bouillon \& F. Busa (Eds.), The language of word meaning (pp. 247-261). Cambridge: Cambridge University Press.

Porto Dapena, J. (2002). Manual de técnica lexicográfica. Madrid: Arco Libros.

Pustejovsky, J. (1995). The Generative Lexicon. Cambridge: MIT Press.

Pustejovsky, J. (2001). Type construction and the logic of concepts. En P. Bouillon \& F. Busa (Eds.), The language of word meaning (pp. 91-123). Cambridge: Cambridge University Press.

Pustejovsky, J. (2011). Coercion in a general theory of argument selection. Linguistics, 49(6), 1401-1431.

Real Academia Española (2003). Diccionario de la lengua española. Vigésima segunda edición [CD-ROM]. Versión 1.0. Madrid: Espasa Calpe.

Ruimy, N., Gola, E. \& Monachini, M. (2001). Lexicography informs lexical semantics: The SIMPLE experience. En P. Bouillon \& F. Busa (Eds.) The language of word meaning (pp. 350-362). Cambridge: Cambridge University Press. 


\section{NOTAS}

1 La cantidad de nombres no coincide necesariamente con la cantidad de definiciones, ya que hay nombres que tienen más de una acepción marcada. En los casos en que las definiciones corresponden a tipos distintos en la ontología, aclaro entre paréntesis y comillas simples el sentido al que me refiero. Por otra parte, de acuerdo con el especialista consultado, algunas de las voces incluidas en el DRAE están desactualizadas. Sin embargo, dado que el objetivo del trabajo es analizar las definiciones y no el lemario, se han incorporado todos los nombres que figuran en el DRAE con las marcas Med. o Anat. y corresponden a uno de los tres tipos seleccionados de la ontología UMLS.

2 La red semántica y el metatesauro se encuentran disponibles de manera gratuita en el siguiente enlace: [http://www.nlm.nih.gov/research/umls/]

3 Hernán Diego Iriarte es médico y docente desde el 2000 de la cátedra de Anatomía, Facultad de Medicina, Universidad de Buenos Aires.

4 Los informantes cuentan con un nivel terciario incompleto o superior y no poseen formación particular en medicina. Han sido elegidos aquellos informantes que presentaban las características mencionadas por considerarse que se adecuan al perfil de un usuario del DRAE, para el cual las definiciones deberían resultar claras.

5 Muchas de las definiciones de los nombres que pertenecen al tipo parte del cuerpo son de la clase que Porto Dapena (2002) denomina 'participativas'. En estas definiciones el núcleo del sintagma no está constituido por un hiperónimo sino por una palabra de sentido general, como 'parte', o por una expresión con sentido distributivo como 'cada uno'. Según Porto Dapena (2002), tampoco constituyen un hiperónimo de la voz definida los complementos de 'parte' o 'cada uno', dado que por ejemplo, una parte de un hueso no es estrictamente un hueso. La noción de qualia dominante permite una explicación y un mejor tratamiento de esta clase de voces. Según Lenci (2001), los principios de la EQ pueden adoptarse para organizar ontologías y el quale constitutivo puede dominar aquellos tipos semánticos que describen sentidos cuya contribución semántica está totalmente determinada por las relaciones meronímicas que contraen con otras unidades léxicas, ya que en estos casos las relaciones de tipo hiperonímico son, de acuerdo con el autor, poco informativas. Consideraré aquí que el QF de estas voces lo constituye la entidad completa de la que forman parte (siguiendo la propuesta de Copestake, 1995), pero que el quale dominante de estas voces el QC.

6 Puede considerarse que la definición de 'alcoholaturo' da cuenta tanto del QA (el medicamento 'se obtiene macerando plantas en alcohol') y del QC (el medicamento 'está constituido por plantas maceradas en alcohol').

\section{* Agradecimientos}

Quisiera agradecer a Paz Battaner por haberme sugerido la idea para este trabajo, a Andreína Adelstein por su lectura de los sucesivos borradores y a Rosa Estopà por su asesoramiento en la elección de la ontología. Asimismo quisiera dar las gracias a los dos evaluadores anónimos que contribuyeron a hacer de este trabajo un trabajo más sólido tanto desde el punto de vista teórico como desde el punto de vista metodológico. Finalmente, agradezco también a Hernán Iriarte por su ayuda en lo que respecta a las cuestiones terminológicas de la medicina y a los informantes que colaboraron en la realización de las encuestas. 